\title{
MODIFICAÇÕES NAS PROPRIEDADES DOS SOLOS DE UMA ÁREA DE MANEJO FLORESTAL NA CHAPADA DO ARARIPE ${ }^{(1)}$
}

\author{
Adriana Oliveira Araújo ${ }^{(2)}$, Luiz Alberto Ribeiro Mendonça ${ }^{(3)}$, Maria Gorethe de Sousa \\ Lima $^{(4)}$, José Valmir Feitosa ${ }^{(4)}$, Fernando José Araújo da Silva ${ }^{(4)}$, Ricardo Luiz Lange

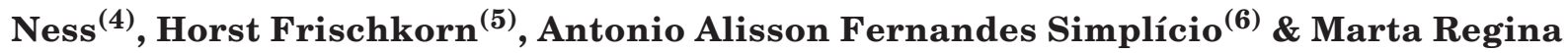 \\ Kerntopf $^{(7)}$
}

\begin{abstract}
RESUMO
A Chapada do Araripe possui grande diversidade ambiental, onde é predominante o Latossolo Vermelho-Amarelo. Nela encontra-se a Floresta Nacional do Araripe (FLONA) e várias unidades de manejo florestal (UMF). $O$ objetivo deste estudo foi utilizar a análise multivariada para obter-se um indicador básico que represente um conjunto de atributos físicos, químicos e biológicos utilizados como indicadores de modificações nas propriedades dos solos de uma UMF. Foram analisados, em triplicata, na profundidade de $0-20 \mathrm{~cm}$, solos de 15 pontos da UMF e quatro da FLONA, onde foram determinados nove atributos utilizados na identificação das peculiaridades de grupos de estado de recuperação dos solos e suas principais diferenças. A análise demonstrou que dois componentes principais respondem por aproximadamente $76 \%$ da variância dos dados: o primeiro é o indicador da qualidade biológica, química e física dos solos; e o segundo, do estado
\end{abstract}

(1) Parte da Dissertação de Mestrado do primeiro autor apresentada ao Programa de Pós-Graduação em Engenharia Agrícola, área de concentração em Manejo e Conservação de Bacias Hidrográficas do Semiárido, da Universidade Federal do Ceará - UFC. Pesquisa financiada com recursos do CNPq (Processo $n^{\circ}$ 50186-4/2009-1) e da FUNCAP (processo ${ }^{\circ}$ BPI-0203-3.07/08). Recebido para publicação em 20 de junho de 2012 e aprovado em 9 de abril de 2013.

${ }^{(2)}$ Doutoranda em Engenharia Agrícola, Departamento de Engenharia Agrícola - DENA, UFC. Campus do Pici, Bloco 804. CEP 60455-760 Fortaleza (CE). Bolsista da FUNCAP. E-mail: adrianasaneamento@yahoo.com.br

(3) Professor Adjunto, DENA, UFC. Campus do Cariri. Av. Tenente Raimundo Rocha, s/n. CEP 63040-360 Juazeiro do Norte (CE). Bolsista de Produtividade do CNPq 2F. E-mail: larm@ufc.br

(4) Professor Adjunto, UFC, Campus do Cariri. E-mail: gorethelima@cariri.ufc.br; valmir_feitosa@yahoo.com.br; fjas@cariri.ufc.br; ness@cariri.ufc.br

(5) Professor Associado, Departamento de Engenharia Hidráulica e Ambiental - DEHA, UFC, Campus do Pici. Caixa Postal 6018. CEP 60451-970 Fortaleza (CE). E-mail: cariri@ufc.br

(6) Mestrando em Solos e Nutrição de Plantas, UFC. Campus do Pici, Bloco 807. CEP 60440-554 Fortaleza (CE). E-mail: alisson_simplicio@yahoo.com.br

(7) Professora Adjunta, Departamento de Química Biológica, Programa de Pós-Graduação em Bioprospecção Molecular, PPBM, Universidade Regional do Cariri - URCA, Campus do Pimenta. Rua Cel. Antônio Luiz, 1161. CEP 63105-000 Crato (CE). E-mail: martaluiz@yahoo.com.br 
de compactação. A análise de agrupamento multivariada, aplicada ao primeiro componente principal, evidenciou a formação de três grupos: um da FLONA e dois contendo amostras da UMF, em diferentes estados de recuperação. A utilização do teste de Tukey em nível de $5 \%$ no agrupamento dos teores de matéria orgânica dos solos apresentou os mesmos grupos da análise de agrupamento multivariada, indicando que esse atributo pode ser utilizado como indicador básico na área de estudo.

Termos de indexação: Floresta Nacional do Araripe, análise de componentes principais, análise de agrupamento, solos degradados.

\title{
SUMMARY: CHANGES IN SOIL PROPERTIES OF A FOREST MANAGEMENT AREA ON THE ARARIPE PLATEAU
}

\begin{abstract}
The Araripe Plateau in the tropic semi-arid region is characterized by great environmental diversity. The Araripe National Forest and several forest management units (FMU) are located in this area with mostly red-yellow Oxisols. The goal of this study was to identify a basic indicator that could represent a set of physical, chemical and biological properties of soils of the Plateau. Multivariate analysis was used to indicate changes in soil properties of a FMU. Soil samples were analyzed in triplicate (0-20 cm layer), from 15 points of the FMU and four points of the National Forest. Nine soil properties were selected to characterize differences between preserved and exploited sites, including those in recovery phases. The results show that two principal components account for approximately $76 \%$ of the variance of the data: the first indicator of biological, chemical and physical soil quality and the second indicator of soil compaction. Multivariate cluster analysis of the first principal component group showed the formation of three sub-groups: the National Forest sub-group and two sub-groups of the FMU in different recovery stages. The Tukey test (5\% significance) applied to the grouping of organic matter content of soils agreed with results from multivariate cluster analysis. This indicates that this organic matter can be used as main indicator of changes in soil properties for the study area.
\end{abstract}

Index terms: National Forest Araripe, principal component analysis, cluster analysis, degraded soils.

\section{INTRODUÇÃO}

A vegetação preservada na Floresta Nacional do Araripe (FLONA), fronteira entre os Estados do Ceará e de Pernambuco, ocupa aproximadamente $5 \%$ da área da Chapada do Araripe, que é de $8.000 \mathrm{~km}^{2}$ (DNPM, 1996; Alves et al., 2011). Essa vegetação exerce grande influência sobre o clima do setor oriental da chapada, com impacto também sobre os recursos hídricos. Fora dessa área preservada, atividades agronômicas e práticas de manejo impõem alterações sobre as qualidades físicas, químicas e biológicas dos solos. Em paralelo, diante desse antropismo, a vegetação tende a apresentar elevado grau de adaptação, notadamente ao déficit hídrico (Araújo, 2010). É necessário, portanto, que as técnicas e estratégias de manejo florestal sejam empregadas para favorecer mecanismos naturais que permitam a reação do ecossistema aos diferentes graus de perturbação impostos (Campello, 1998). Nesse sentido, o manejo florestal monitorado é capaz de minimizar impactos ambientais produzidos nas propriedades físicas, químicas e biológicas dos solos antropizados, o que possibilita a recomposição dos teores de matéria orgânica do solo e da disponibilidade de nutrientes. Porém, a intensidade dessas modificações varia com as condições edafoclimáticas, a natureza do solo e os usos e manejos adotados (Centurion et al., 2001; Araújo et al., 2004; Figueiredo et al., 2008).

Modificações nas propriedades dos solos podem ser acompanhadas por meio do monitoramento de atributos físicos, químicos e biológicos. Dentre esses, recomenda-se monitorar os que indicam mudanças em médio prazo, com destaque para: densidade, macro e microporosidade, matéria orgânica, umidade, capacidade de infiltração de água, resistência à penetração e densidade de indivíduos da macrofauna edáfica (Araújo et al., 2004; Oliveira et al., 2004; Souza et al., 2005).

Entretanto, as determinações desses atributos dos solos podem ser caras e demandam tempo. Assim, o monitoramento regular em regiões carentes de suporte técnico especializado é pouco eficiente, tornando imprescindível a realização de investigações que permitam compreender a função desempenhada por cada atributo analisado, de forma que se possam selecionar os que melhor reflitam as condições de manejo do solo. 
A análise multivariada de dados pode contribuir para compreensão da função desempenhada por cada atributo analisado, podendo identificar qual ou quais devem ser utilizados como indicadores básicos. Esses possibilitam melhor entendimento da dinâmica de alterações da estrutura do solo antropizado, com identificação de indicadores básicos, redução do número de análises e otimização do monitoramento contínuo.

O objetivo deste estudo é determinar indicadores de atributos físicos, químicos e biológicos que expliquem modificações nas propriedades dos solos de uma área de manejo florestal, por meio da análise multivariada de dados.

\section{MATERIAL E MÉTODOS}

O estudo foi realizado na Floresta Nacional do Araripe (FLONA) e na unidade de manejo florestal (UMF) das Fazendas Pau D'árco e Bonfim, localizadas no setor oriental da Chapada do Araripe, extremo sul do Estado do Ceará (9183896 - 9199039 N e 435987 460703 S, Zona 24S, SAD-69 datum) (Figura 1). O clima da região é do tipo Aw', tropical chuvoso, com temperatura média máxima de $34^{\circ} \mathrm{C}$, média mínima de $18{ }^{\circ} \mathrm{C}$, umidade relativa do ar média máxima de $80 \%$ e média mínima de $49 \%$ (INMET, 1993). A precipitação pluvial média anual é de $1.033 \mathrm{~mm}$, com período chuvoso entre janeiro e maio e seco entre setembro e novembro (DNPM, 1996).

A FLONA, com área de aproximadamente 383 $\mathrm{km}^{2}$, foi a primeira Unidade de Conservação federal do Brasil, criada pelo Decreto 9.226, de 02 de maio de 1946, com o objetivo de conservar os recursos florestais para manter centenas de nascentes perenes que irrigam os vales. Ela é gerida pelo ICMBio e é constituída, predominantemente, de floresta úmida semiperenifólia, cerradão e cerrado (Carvalho et al., 1999; Embrapa, 2006; Alves et al., 2011). A UMF do estudo tem área de aproximadamente $15 \mathrm{~km}^{2}$, está localizada na zona de entorno da FLONA e é constituída de cerrado. Os solos são do tipo Latossolo Vermelho-Amarelo, de textura média a argilosa em todas as formações florestais. As áreas de estudo encontram-se em altitude de aproximadamente $1.000 \mathrm{~m}$, em relevo tabular plano; a FLONA, porém, encontra-se limitada por escarpas abruptas em direção ao Vale do Cariri, com desnível de aproximadamente $400 \mathrm{~m}$.

AUMF foi implantada em 2002, com a área dividida em 11 talhões (Figura 1), que são explorados individualmente em sequência anual, para fornecer lenha a uma indústria cerâmica. Durante a exploração, ocorre trânsito de pessoas e eventualmente de máquinas, principalmente nas margens dos talhões explorados. Antes da implantação, a partir de 1974, a área havia sido degradada para plantio de café e outras culturas, além de abrigar aproximadamente 52 famílias, que sobreviviam da caça e da produção clandestina de carvão vegetal.

Foram analisadas amostras de solos de quatro localidades não alteradas da FLONA, consideradas como controle e identificadas como F1, F2, F3 e F4, e 15 localidades da UMF, identificadas como área de reserva legal R1 (A e B) e talhões T1, T2, T3 (A e B), explorados há pelo menos um ano; T4 (A a D), com exploração em andamento; T5, T6 e T7, não explorados (Figura 1). Nos talhões não explorados T8 a T11 e na reserva legal R2, não foram feitas coletas por causa da dificuldade de acesso.

Nos solos de cada local de amostragem da FLONA e UMF foram determinados, em triplicatas, na profundidade de $0-20 \mathrm{~cm}$, os seguintes atributos biológicos, químicos e físicos: densidade da macrofauna edáfica (DMF), teor de matéria orgânica do solo $(\mathrm{MOS})$, umidade $(\mathrm{U})$, resistência à penetração $(\mathrm{RP})$, macro (Ma) e microporosidade (Mi) e capacidade de infiltração básica (Ic). Os três primeiros foram definidos em campanhas realizadas no período seco,

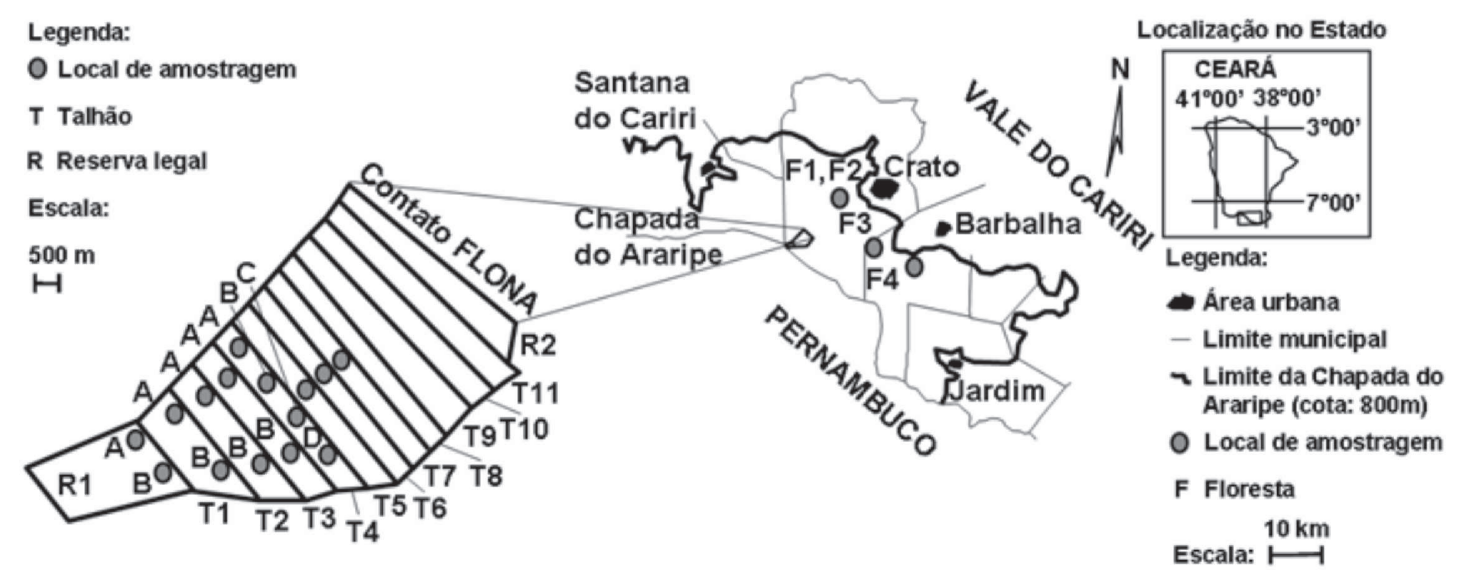

Figura 1. Locais de amostragens na Unidade de Manejo Florestal estudada (R e T) e na Floresta Nacional do Araripe (F). 
em novembro e dezembro de 2008 , e no chuvoso, em abril de 2009. Os demais foram estabelecidos em uma única campanha no período seco, em outubro de 2008.

A DMF foi determinada conforme Harada \& Bandeira (1998) e adaptada por Baretta (2003), com animais obtidos por catação manual em solos coletados com uso de amostrador de aço de $25 \times 25 \times 20 \mathrm{~cm}$.

Os teores de MOS foram determinados por ignição em mufla a $550{ }^{\circ} \mathrm{C}$ e os de umidade, pelo método gravimétrico, de acordo com Embrapa (1997).

As RPs foram estipuladas com uso do penetrômetro de impacto modelo Stolf, de massa de impacto de $4 \mathrm{~kg}$ e altura de queda livre de $40 \mathrm{~cm}$ (Stolf et al., 1983).

As Ma e Mi foram determinadas por mesa de tensão de sucção de $60 \mathrm{~cm}$ de coluna d'água (Kiehl, 1979; Lima \& Silva, 2008), enquanto a Ic foi estimada por ajuste à equação de Horton, da curva taxa de infiltração no tempo, obtida com infiltrômetro de duplo cilindro concêntrico de carga hidráulica variável (Hillel, 1998).

Em análise estatística inicial, construiu-se uma matriz de correlação de Pearson, para avaliação do grau de associação entre atributos. Em seguida, aplicou-se a técnica da análise fatorial pelo método das componentes principais, utilizando-se a rotação varimax normalizada. Tal abordagem buscou descrever as relações de covariância entre os atributos correlacionados, com base em um pequeno número de quantidades aleatórias chamadas fatores, além de evidenciar, por meio de comunalidades, o quanto cada atributo explica cada fator (Hoffmann, 1992; Manly, 1998).

Para verificar a adequação dos dados, a análise fatorial utilizou-se o índice KMO (Kaiser-Meyer-Olkin Measure of Sampling Adequacy) e o teste estatístico de esfericidade de Bartlett, que testa a hipótese nula de que as variáveis analisadas não são correlacionadas (Hair Jr. et al., 1998). Em seguida, procedeu-se à análise de agrupamento multivariada, visando agrupar amostras a partir do maior número de atributos explicados por um único fator da análise fatorial. Nessa técnica, utilizou-se o método do "vizinho mais distante" (furthest neighbor) como critério hierárquico de agrupamento, com medida de similaridade dada pela "distância euclidiana quadrada" (squared euclidean distance).

Após o agrupamento, os atributos padronizados foram submetidos à análise de variância One-Way ANOVA, dentro de cada grupo gerado, para verificar se houve a formação de um conjunto estável de grupos (Bussab et al., 1990). Essas análises estatísticas foram processadas no software SPSS Statistics, versão 17.0.

\section{RESULTADOS E DISCUSSÃO}

No quadro 1, é apresentado um sumário dos resultados dos atributos analisados. As médias de MOS por amostra não apresentaram diferenças significativas entre os períodos chuvoso e seco, com base no teste de Tukey, em nível de $5 \%$, sendo, portanto, representados em uma única coluna pelos valores médios entre esses períodos.

De acordo com os valores médios, na UMF (R e T) observou-se menor DMF nos períodos chuvoso e seco, quando comparada à área da FLONA $(\mathrm{F})$, apesar de os talhões não explorados R1, T5 e T6 apresentarem valores elevados no período seco. Esse fato pode ser explicado pela elevada presença da ordem taxonômica Isoptera (cupim), que se adaptam às condições edafoclimáticas desfavoráveis predominantes nesse período. Nessa área de manejo, também foram encontrados menores teores de MOS e U nos períodos chuvoso e seco, além de serem observados, por amostra, teores de U no período chuvoso inferiores aos da área da FLONA no período seco. Para esses atributos, os coeficientes de variação foram expressivamente superiores para a área da UMF, indicando maior heterogeneidade dos dados.

Esses resultados sugerem que a utilização de sistemas de manejo florestal pode acarretar mudanças na densidade da biota dos solos, em razão da redução no fornecimento de material orgânico e exposição dos solos às intempéries, com variações extremas de temperatura e umidade, desfavorecendo as condições bioedáficas. Os resultados corroboram com Reatto et al. (2009), que estudaram o efeito de diferentes tipos de manejo na modificação da densidade da macrofauna edáfica.

A RP foi maior na área da UMF, quando comparada à área da FLONA, indicando maior compactação dos solos. Essa compactação deve-se às pressões exercidas sobre o solo, por causa do trânsito de máquinas e pessoas durante a exploração e pela exposição desse ao impacto direto das gotas de chuva. Ainda na área da UMF, verificou-se elevação na Mi e, conversivamente, redução na Ma e Ic. Esses resultados sugerem que a compactação das áreas manejadas é capaz de produzir alterações na estrutura dos solos. Esses achados também corroboram com Argenton et al. (2005), Santos et al. (2006), Lima et al. (2007) e Alves et al. (2007), que estudaram o comportamento de atributos da estrutura de solos submetidos a diferentes tipos de manejo. Os coeficientes de variação desses atributos analisados também foram expressivamente superiores para a área da UMF.

No quadro 2, encontra-se a matriz de correlação para os atributos analisados. Observou-se que 29 dos coeficientes de correlação, $81 \%$ do total de pares, apresentaram valores significativos. Desses, aproximadamente $66 \%$ encontraram-se no intervalo $0,6 \leq|\mathrm{r}|<0,9$, caracterizados, segundo CallegariJacques (2003), como de correlação forte. O restante (34\%) encontrou-se no intervalo $0,3 \leq|\mathrm{r}|<0,6$, caracterizados como de correlação moderada.

A matriz de correlação evidenciou que a DMFc é positivamente correlacionada com a MOS, Uc, Us, 
Ma e Ic. Já a DMFs apresentou-se positivamente correlacionada apenas com a MOS e Us. Porém, tanto a DMFc quanto a DMFs revelou correlação negativa com a Mi. Esses resultados indicaram que a DMF está relacionada à umidade e disponibilidade de alimento, que também estão diretamente relacionadas à MOS e inversamente à Mi. Além do mais, as estruturas biogênicas (galerias, ninhos, câmaras e

Quadro 1. Atributos biológicos, químicos e físicos das áreas de floresta preservada e de manejo florestal na Chapada do Araripe, na profundidade de $0-20 \mathrm{~cm}$

\begin{tabular}{|c|c|c|c|c|c|c|c|c|c|}
\hline Amostra & DMFc & DMFs & MOS & Uc & Us & $\mathbf{R P}$ & Ma & Mi & Ic \\
\hline & \multicolumn{2}{|c|}{ indivíduos $\mathrm{m}^{-2}$} & \multicolumn{3}{|c|}{$\mathrm{g} \mathrm{kg}^{-1}$} & impactos $\mathrm{dm}^{-1}$ & \multicolumn{2}{|c|}{$-m^{3} m^{-3}$} & $\mathrm{~cm} \mathrm{~min} \min ^{-1}$ \\
\hline $\mathrm{R} 1 \mathrm{~A}$ & 112,0 & 528,0 & 6,8 & 12,5 & 11,0 & 1,3 & 15,6 & 35,9 & 1,7 \\
\hline $\mathrm{R} 1 \mathrm{~B}$ & 80,0 & 176,0 & 6,6 & 14,3 & 14,0 & 0,9 & 6,3 & 36,0 & 2,2 \\
\hline $\mathrm{T} 1 \mathrm{~A}$ & 112,0 & 16,0 & 2,9 & 12,2 & 5,0 & 2,3 & 4,5 & 58,2 & 2,5 \\
\hline $\mathrm{T} 1 \mathrm{~B}$ & 96,0 & 32,0 & 1,9 & 10,3 & 7,0 & 3,6 & 10,1 & 51,1 & 2,5 \\
\hline $\mathrm{T} 2 \mathrm{~A}$ & 176,0 & 64,0 & 2,9 & 17,0 & 8,0 & 3,0 & 4,2 & 53,8 & 2,6 \\
\hline $\mathrm{T} 2 \mathrm{~B}$ & 128,0 & 0,0 & 1,8 & 13,1 & 8,0 & 1,0 & 14,9 & 46,6 & 0,4 \\
\hline T3A & 240,0 & 48,0 & 1,9 & 11,3 & 8,0 & 2,0 & 14,8 & 32,6 & 1,1 \\
\hline T3B & 32,0 & 112,0 & 2,6 & 12,9 & 8,0 & 2,0 & 16,8 & 46,5 & 2,3 \\
\hline $\mathrm{T} 4 \mathrm{~A}$ & 176,0 & 32,0 & 7,1 & 14,3 & 11,0 & 1,1 & 18,3 & 38,8 & 2,2 \\
\hline $\mathrm{T} 4 \mathrm{~B}$ & 192,0 & 64,0 & 7,4 & 10,9 & 7,0 & 1,2 & 10,5 & 46,9 & 2,5 \\
\hline $\mathrm{T} 4 \mathrm{C}$ & 144,0 & 96,0 & 5,9 & 14,6 & 8,0 & 1,6 & 15,0 & 44,7 & 2,0 \\
\hline $\mathrm{T} 4 \mathrm{D}$ & 192,0 & 16,0 & 8,3 & 14,3 & 11,0 & 1,5 & 1,2 & 47,2 & 1,2 \\
\hline T5 & 272,0 & 208,0 & 7,2 & 13,1 & 9,0 & 2,2 & 19,2 & 38,5 & 1,5 \\
\hline $\mathrm{T} 6$ & 192,0 & 288,0 & 7,1 & 15,4 & 9,0 & 2,0 & 14,1 & 39,1 & 2,0 \\
\hline $\mathrm{T} 7$ & 256,0 & 32,0 & 7,7 & 13,2 & 9,0 & 1,8 & 7,1 & 48,9 & 3,2 \\
\hline Média & 160,0 & 114,1 & 5,2 & 13,3 & 8,9 & 1,8 & 11,5 & 44,3 & 2,0 \\
\hline $\mathrm{CV}(\%)$ & 42,4 & 123,1 & 48,0 & 13,3 & 24,4 & 40,9 & 49,2 & 16,4 & 35,6 \\
\hline F1 & 320,0 & 160,0 & 13,2 & 29,1 & 13,0 & 0,7 & 31,7 & 31,3 & 3,5 \\
\hline F2 & 544,0 & 128,0 & 12,2 & 28,6 & 19,0 & 0,9 & 29,7 & 31,4 & 3,6 \\
\hline F3 & 336,0 & 224,0 & 12,5 & 26,6 & 20,0 & 0,8 & 22,4 & 27,4 & 3,8 \\
\hline $\mathrm{F} 4$ & 544,0 & 272,0 & 12,0 & 24,6 & 20,0 & 0,6 & 20,2 & 30,9 & 3,5 \\
\hline Média & 436,0 & 196,0 & 12,5 & 27,2 & 18,0 & 0,8 & 26,0 & 30,3 & 3,6 \\
\hline $\mathrm{CV}(\%)$ & 28,6 & 32,9 & 4,2 & 7,6 & 18,7 & 17,2 & 21,4 & 6,3 & 3,9 \\
\hline
\end{tabular}

DMF: densidade da macrofauna edáfica; c e s: índices referentes aos períodos chuvoso e seco, respectivamente; MOS: matéria orgânica dos solos; U: umidade; RP: resistência mecânica à penetração; Ma: macroporosidade x $10^{2}$; Mi: microporosidade x $10^{2}$; Ic: capacidade de infiltração básica; e CV: coeficiente de variação.

Quadro 2. Matriz de correlação dos atributos biológicos, químicos e físicos dos solos das áreas de floresta preservada e de manejo florestal na Chapada do Araripe

\begin{tabular}{|c|c|c|c|c|c|c|c|c|c|}
\hline Atributo & DMFc & DMFs & MOS & Uc & Us & $\mathbf{R P}$ & Ma & $\mathbf{M i}$ & Ic \\
\hline DMFc & 1,00 & & & & & & & & \\
\hline DMFs & 0,18 & 1,00 & & & & & & & \\
\hline MOS & $0,76^{*}$ & $0,40 *$ & 1,00 & & & & & & \\
\hline $\mathrm{Uc}$ & $0,78^{*}$ & 0,25 & $0,82^{*}$ & 1,00 & & & & & \\
\hline Us & $0,78^{*}$ & $0,42^{*}$ & $0,83^{*}$ & $0,83^{*}$ & 1,00 & & & & \\
\hline $\mathrm{RP}$ & $-0,46^{*}$ & $-0,29$ & $-0,69 *$ & $-0,56^{*}$ & $-0,67^{*}$ & 1,00 & & & \\
\hline $\mathrm{Ma}$ & $0,60^{*}$ & 0,34 & $0,59^{*}$ & $0,72^{*}$ & $0,59^{*}$ & $-0,51^{*}$ & 1,00 & & \\
\hline $\mathrm{Mi}$ & $-0,61^{*}$ & $-0,55^{*}$ & $-0,68^{*}$ & $-0,64^{*}$ & $-0,80^{*}$ & $0,67^{*}$ & $-0,75^{*}$ & 1,00 & \\
\hline Ic & $0,57^{*}$ & 0,15 & $0,66^{*}$ & $0,71^{*}$ & $0,60^{*}$ & $-0,22$ & $0,41^{*}$ & $-0,29$ & 1,00 \\
\hline
\end{tabular}

* valores significativos com p<0,05; DMF: densidade da macrofauna edáfica; c e s: índices referentes aos períodos chuvoso e seco, respectivamente; MOS: matéria orgânica dos solos; U: umidade; RP: resistência mecânica à penetração; Ma: macroporosidade x $10^{2}$; Mi: microporosidade x $10^{2}$; Ic: capacidade de infiltração básica. 
pellets fecais) produzidas pela macrofauna edáfica, associadas ao sistema radicular da vegetação, são capazes de elevar a Ic e manter a estrutura dos solos, preservando os macroporos e reduzindo os microporos. Nesse contexto, observa-se que alterações produzidas na estrutura dos solos por ações antrópicas, capazes de interferir principalmente na Ma e MOS, podem resultar em forte impacto na DMF. Resultados semelhantes foram observados por Blanchart (1992), Lavelle \& Spain (2001), Barros et al. (2001) e Silva et al. (2006), que verificaram a sensibilidade da macrofauna edáfica ao uso e manejo da vegetação e do solo.

A RP foi positivamente correlacionada com a Mi e negativamente com a Ma, com a DMFc e com os teores de MOS, Uc e Us. Esses resultados indicaram que o processo de compactação do solo altera a distribuição dos poros, aumentando a Mi e reduzindo a Ma. Segundo Albuquerque et al. (2001), Merlim et al. (2005) e Argenton et al. (2005), essas interferências, dependendo do nível de compactação, podem restringir a aeração e o crescimento das raízes, influindo severamente na DMF. Essa parece ser uma das principais causas de degradação de áreas manejadas. Também, áreas compactadas e com reduzida cobertura do solo por serapilheira de detritos vegetais podem causar decréscimo nos teores de MOS e de U e aumento na temperatura da camada superficial exposta, contribuindo com redução na DMF, mantendo o solo desprotegido contra a degradação.

A partir dessa análise, procedeu-se à análise fatorial de modo a agregar as observações feitas para os nove atributos, com vistas a entender o papel de cada um na dinâmica de alterações na estrutura dos solos, causadas por ações antrópicas nas áreas de manejo florestal de vegetação nativa.

O índice $K M O$, obtido para o conjunto de atributos analisados, foi de 0,766 e o teste estatístico de esfericidade de Bartlett evidenciou-se significativo a $p<0,01$, indicando que os fatores a serem encontrados na Análise Fatorial podem descrever adequadamente a variação dos dados originais.

No quadro 3, estão contidos os resultados da análise de componentes principais, em que foram extraídos dois fatores com autovalores superiores a um. Esses foram submetidos à rotação varimax normalizada, de onde se obteve para o primeiro uma contribuição para explicação da variância total dos atributos de aproximadamente $44 \%$ e, para o segundo, de aproximadamente $32 \%$. Esse resultado indicou que esses dois fatores respondem por aproximadamente 76 \% da variância dos dados.

Os atributos que mais se associaram com os componentes principais (fatores) apresentaram cargas fatoriais superiores a 0,6 (valores absolutos). As maiores cargas fatoriais indicaram maiores coeficientes de correlação entre cada fator e cada um dos nove atributos analisados. As cargas fatoriais ligeiramente inferiores a 0,6 representaram atributos que se associaram com os fatores de forma moderada.
As comunalidades indicaram que entre 80 e $90 \%$ da variância dos atributos MOS, Uc, Us e Mi foram explicadas pelos dois fatores. Já os atributos Ic e DMFc tiveram entre 70 e $80 \%$ da variabilidade explicada; e os RP, Ma e DMFs, entre 60 e $70 \%$.

A primeira componente principal (fator 1) encontrase fortemente correlacionada com atributos indicadores da qualidade biológica, química e física dos solos: DMFc, MOS, Uc, Us e Ic. De forma moderada, correlaciona-se também com a Ma.

Os atributos explicados pelo fator 1 mantêm-se em estrito relacionamento, pois em solos de vegetação preservada há um aporte de matéria orgânica, conservando a umidade, aumentando a capacidade de absorção e infiltração de água, reduzindo o risco de erosão e estimulando a atividade da macrofauna edáfica (Muschler, 2000; Barbera-Castillo, 2001).

Há a hipótese de que a abundância da macrofauna edáfica pode ser usada como indicador da qualidade dos solos, por ser muito sensível às modificações produzidas por diferentes tipos de manejo, conforme destaca Socarrás (1998). Para Lavelle \& Spain (2001), a comunidade da macrofauna edáfica pode ser facilmente influenciada por modificações produzidas por diversos tipos de manejos, em razão da destruição de habitats, da exposição aos predadores e das condições edafoclimáticas desfavoráveis produzidas por expressivas variações de temperatura e umidade.

A segunda componente principal (fator 2) correlaciona-se fortemente com atributos indicadores do estado da compactação do solo (Camargo \& Alleoni, 1997): RP, Mi e DMFs. Esse fator também se correlaciona de forma moderada com a variável Ma,

Quadro 3. Cargas fatoriais, comunalidades e variância explicada na análise fatorial dos nove atributos analisados, após a rotação pelo método varimax

\begin{tabular}{cccc}
\hline \multirow{2}{*}{ Atributo } & \multicolumn{2}{c}{ Carga fatorial } & \multirow{2}{*}{ Comunalidade } \\
\cline { 2 - 3 } & Fator $\mathbf{1}$ & Fator $\mathbf{2}$ & \\
\hline DMFc & 0,826 & 0,291 & 0,767 \\
DMFs & $-0,039$ & 0,797 & 0,637 \\
MOS & 0,773 & 0,497 & 0,845 \\
Uc & 0,876 & 0,351 & 0,891 \\
Us & 0,739 & 0,566 & 0,867 \\
RP & $-0,390$ & $-0,670$ & 0,600 \\
Ma & 0,541 & 0,573 & 0,622 \\
Mi & $-0,415$ & $-0,837$ & 0,872 \\
Ic & 0,866 & $-0,046$ & 0,751 \\
\% da variância & 44,03 & 32,12 & \\
explicada pelo fator & & & \\
\hline
\end{tabular}

DMF: densidade da macrofauna edáfica; c e s: índices referentes aos períodos chuvoso e seco, respectivamente; MOS: matéria orgânica dos solos; U: umidade; RP: resistência mecânica à penetração; Ma: macroporosidade; Mi: microporosidade; Ic: capacidade de infiltração básica. 


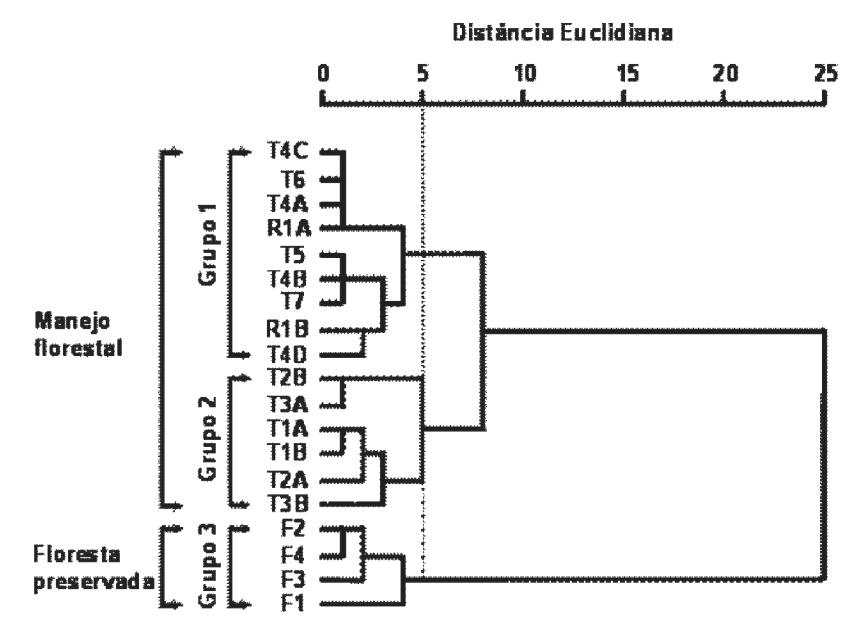

Figura 2. Dendrograma resultante da análise de agrupamento das variáveis explicadas pelo fator 1 da análise fatorial.

além de contribuir para explicar aproximadamente 32 \% da variância total. Os resultados sugeriram que a falta de controle no manejo da vegetação e do solo leva a modificações na estrutura desse, com destaque para compactação, elevação da microporosidade e redução da macroporosidade, interferindo sobre o desenvolvimento radicular da vegetação e a atividade biológica. Isso é mais relevante no período seco, quando ocorre déficit de umidade no solo antropizado, encontrando-se esse submetido a elevadas temperaturas (Dias Jr. \& Estanislau, 1999; Souza \& Alves, 2003).

A técnica de análise de agrupamento multivariada, aplicada aos atributos explicados pelo fator 1 da análise fatorial, agrupou as amostras das áreas de floresta preservada e de manejo florestal com características semelhantes (Figura 2). Os grupos obtidos e os atributos que os geraram foram submetidos à análise de variância One-Way ANOVA e apresentaram níveis de significância inferiores a $5 \%$, indicando a formação de um conjunto relativamente estável de grupos.

Para a distância de corte em cinco, observou-se a formação de três grupos homogêneos. Os Grupos 1 e 2 continham amostras da área da UMF, enquanto o Grupo 3, da FLONA. O Grupo 1 reuniu amostras da reserva legal (R1), do talhão com exploração em andamento (T4) e dos talhões ainda não explorados (T5, T6 e T7); e o Grupo 2, amostras dos talhões explorados há pelo menos um ano (T1, T2 e T3).

Considerando a MOS como possível indicador básico da qualidade dos solos (Doran \& Parkin, 1994; Alves \& Cabeda, 1999), procedeu-se ao ordenamento de grupos pela observação de diferenças significativas entre as médias, utilizando teste de Tukey a $5 \%$. Observou-se por meio desse procedimento que os agrupamentos foram os mesmos obtidos pela técnica de análise de agrupamento multivariada. O grupo de amostras dos talhões explorados há pelo menos um ano apresentou menor teor de $\operatorname{MOS}\left(2,3 \mathrm{~g} \mathrm{~kg}^{-1}\right)$ do que os demais talhões $\left(7,1 \mathrm{~g} \mathrm{~kg}^{-1}\right)$, que foram reunidos em outro grupo, e do que o das áreas de floresta preservada na FLONA (12,5 $\left.\mathrm{g} \mathrm{kg}^{-1}\right)$, indicando o efeito da exposição dos solos desprotegidos às intempéries. Nesse contexto, observou-se que o teor de MOS refletiu nas variações ocorridas nos atributos indicadores da qualidade física e biológica dos solos das áreas estudadas, explicadas pelo fator 1 da análise fatorial, por apresentar os mesmos agrupamentos obtidos pela técnica de análise de agrupamento multivariada.

Agrupamentos semelhantes também foram observados por Mendonça et al. (2009), utilizando a mesma metodologia da análise de agrupamento univariada adotada nesta pesquisa, porém numa área bem mais extensa da Chapada do Araripe. Esses autores notaram a formação de quatro grandes grupos. Nesse caso, o grupo de maior teor de MOS, constituído de amostras de solos coletadas em áreas de floresta preservada e de cerrado desmatado e abandonado em recuperação, apresentou maior teor de U e Ic; e o de menor teor de MOS, composto de amostras de áreas de manejo de eucalipto em diversas fases de crescimento, de manejo de vegetação nativa com clareiras, de áreas de cultivos abandonados e de cerrado desmatado com solo exposto e indícios de processos erosivos, também apresentou menor teor de U e Ic.

\section{CONCLUSÕES}

1. A análise fatorial e a análise de agrupamento multivariada contribuíram com o entendimento das modificações nas propriedades dos solos da área de manejo florestal.

2. A análise fatorial aplicada a nove atributos dos solos evidenciou que dois componentes respondem por aproximadamente $76 \%$ da variância dos dados: o primeiro como indicador da qualidade biológica, química e física dos solos, representado por DMFc, MOS, Uc, Us, Ic e Ma; e o segundo, como indicador do estado de compactação, representado por RP, Mi e DMFs.

3. A análise de agrupamento multivariada, aplicada aos atributos do primeiro componente da análise fatorial, evidenciou a formação de três grupos: um com amostras da Floresta Nacional do Araripe e dois com amostras da unidade de manejo florestal em diferentes estados de recuperação.

4. O mesmo agrupamento da análise multivariada foi encontrado, utilizando-se apenas o teste de Tukey, a $5 \%$, aplicado aos teores de MOS, indicando que esse atributo pode ser utilizado como indicador básico, capaz de explicar as alterações que ocorrem nos solos antropizados da área estudada, contribuindo com a redução do número de análises e com a otimização do monitoramento contínuo. 


\section{AGRADECIMENTOS}

Ao CNPq e à FUNCAP, pelo apoio financeiro; ao IBAMA, à COGERH, à FATEC Cariri, à UFC, pelo apoio logístico; e aos proprietários das Fazendas Pau d'Árco e Bonfim.

\section{LITERATURA CITADA}

ALBUQUERQUE, J.A.; SANGOI, L. \& ENDER, M. Efeito da integração lavoura pecuária nas propriedades físicas do solo e características da cultura do milho. R. Bras. Ci. Solo, 25:717-723, 2001.

ALVES, C.C.E.; BEZERRA, L.M.A. \& MATIAS, A.C.C. A importância da conservação/preservação ambiental da Floresta Nacional do Araripe para a região do Cariri Ceará/Brasil. R. Geogr. Am. Central, Número Especial EGAL: 1-10, 2011.

ALVES, M.C. \& CABEDA, M.S.V. Infiltração em um Podzólico Vermelho-Escuro sob dois métodos de preparo, usando chuva simulada com duas intensidades. R. Bras. Ci. Solo, 15:139-145, 1999.

ALVES, M.C.; SUZUKI, L.G.A. \& SUZUKI, L.E.A.S. Densidade do solo e infiltração de água como indicadores da qualidade física de um latossolo vermelho distrófico em recuperação. R. Bras. Ci. Solo, 31:617-625, 2007.

ARAÚJO, A.O. Avaliação de propriedades físicas dos solos e da macrofauna edáfica em áreas submetidas a manejo florestal de vegetação nativa na Chapada do Araripe. Fortaleza, Universidade Federal do Ceará, 2010. 76p. (Dissertação de Mestrado)

ARAÚJO, M.A.; TORMENA, C.A. \& SILVA, A.P. Propriedades físicas de um Latossolo Vermelho distrófico cultivado e sob Mata Nativa. R. Bras. Ci. Solo, 28:337-345, 2004.

ARGENTON, J.; ALBUQUERQUE, J.A.; BAYER, C. \& WILDNER, L.P. Comportamento de atributos relacionados com a forma da estrutura de Latossolo Vermelho sob sistemas de preparo e plantas de cobertura. R. Bras. Ci. Solo, 29:425-435, 2005.

BARBERA-CASTILLO, N.M. Diversidad de especies de hormigas en sistemas agroforestales contrastantes de café, em Turrialba, Costa Rica. Turrialba, Costa Rica, Centro Agronómico Tropical de Investigación y Enseñanza, 2001. 99p. (Dissertação de Mestrado)

BARETTA, D. Atributos biológicos do solo em diferentes agroecossistemas na região sul do Brasil. Lages, Universidade do Estado de Santa Catarina, 2003. 123p. (Dissertação de Mestrado)

BARROS, E.; CURMI, P.; HALLAIRE, V.; CHAUVEL, A. \& LAVELLE, P. The role of macrofauna in the transformation and reversibility of soil structure of an Oxisol in the process of forest to pasture conversion. Geoderma, 100:193-213, 2001.

BLANCHART, E. Role of earthworms in the restoration of the macroaggregate structure of a de-structured soil under field conditions. Soil Biol. Biochem., 24:1587-1594, 1992.
BUSSAB, W.O.; MIAZAKI, E.S. \& ANDRADE, D.F. Introdução à análise de agrupamentos. São Paulo, IME/USP, 1990. $105 p$.

CALLEGARI-JACQUES, S.M. Bioestatística: Princípios e aplicações. Porto Alegre, Artemed, 2003. 255p.

CAMARGO, O.A. \& ALLEONI, L.R.F. Compactação do solo e o desenvolvimento das plantas. Piracicaba, Escola Superior de Agricultura Luiz de Queiroz, 1997. 132p.

CAMPELLO, E.F.C. Sucessão vegetal na recuperação de áreas degradadas. In: DIAS, L.E. \& GRIFFITH, J.J., eds. Recuperação de áreas degradadas. Viçosa, MG, Universidade Federal de Viçosa, 1998. p.183-196.

CARVALHO, O.L.; AQUINO, B.F.; FRISCHKORN, H.; AQUINO, M.D. \& FONTENELE, R.E.S. Tecnologia agrícola e de conservação ambiental para o topo da Chapada do Araripe. Fortaleza, BNB/FINEP - ACEP, 1999. p.232. (Relatório Técnico Final)

CENTURION, J.F.; CARDOSO, J.P. \& NATALE, W. Efeito de formas de manejo em algumas propriedades físicas e químicas de um Latossolo Vermelho em diferentes agroecossistemas. R. Bras. Eng. Agríc. Amb., 5:254-258, 2001.

DEPARTAMENTO NACIONAL DA PRODUÇÃO MINERAL DNPM. Projeto de avaliação hidrogeológica da bacia sedimentar do Araripe. Recife: 1996.

DIAS JR., M.S. \& ESTANISLAU, W.T. Grau de compactação e retenção de água de Latossolos submetidos a diferentes sistemas de manejo. R. Bras. Ci. Solo, 23:45-51, 1999.

DORAN, J.W. \& PARKIN, T.B. Defining and assessing soil quality. In: DORAN, J.W.; COLEMAN, D.C.; BELDICEK, D.F. \& STEWART, B.A., orgs. Defining soil quality for a sustainable environment. Madison, Soil Science Society of America, 1994. p.3-21.

EMPRESA BRASILEIRA DE PESQUISA AGROPECUÁRIA EMBRAPA. Centro Nacional de Pesquisa do Solo. Manual de métodos de análises de solo. 2.ed. Rio de Janeiro, 1997. $212 p$.

EMPRESA BRASILEIRA DE PESQUISA AGROPECUÁRIA EMBRAPA. Centro Nacional de Pesquisa do Solo. Sistema brasileiro de classificação de solos. 2.ed. Rio de Janeiro, 2006. 306p.

FIGUEIREDO, C.C.; RAMOS, M.L.G. \& TOSTES, R. Propriedades físicas e matéria orgânica de um Latossolo vermelho sob sistemas de manejo e cerrado nativo. Biosc. J., 24:24-30, 2008.

HAIR JR., J.F.; ANDERSON, R.E.; TATHAN, R.L. \& BLACK, W.C. Multivariate data analysis. New Jersey, Prentice Hall, 1998. 928p.

HARADA, A.Y. \& BANDEIRA, A.G. Densidade e distribuição vertical de macroinvertebrados em solos argilosos e arenosos na Amazônia central. Acta Amaz., 28:191-204, 1998.

HILLEL, D. Environmental soil physics. New York, Academic Press, 1998. 770p. 
HOFFMANN, R. Componentes principais e análise fatorial. Piracicaba, Escola Superior de Agricultura Luiz de Queiroz, 1992. 25p. (Série Didática, 76)

INSTITUTO NACIONAL DE METEOROLOGIA - INMET. Normais climatológicas: 1961-1990. Brasília, 1993. 84p.

KIEHL, E.J. Manual de edafologia: relação solo-planta. São Paulo, Agronômica Ceres, 1979. 264p.

LAVELLE, P. \& SPAIN, A.V. Soil ecology. Dordrecht, Kluwer Academic Publication, 2001. 654p.

LIMA, C.G.R.; CARVALHO, M.P.; MELLO, L.M.M. \& LIMA, R.C. Correlação linear e espacial entre produtividade de forragem, a porosidade total e a densidade do solo de Pereira Barreto (SP). R. Bras. Ci. Solo, 31:1233-1244, 2007.

LIMA, H.V. \& SILVA, A.P. Mesa de tensão com areia: Procedimentos para montagem e validação. R. Bras. Ci. Solo, 32:2209-2214, 2008.

MANLY, B.F.J. Multivariate statistical methods: A primer. 2.ed. London, Chapman \& Hall, 1998. 215p.

MENDONÇA, L.A.R.; VÁSQUEZ, M.A.N.; FEITOSA, J.V.; OLIVEIRA, J.F.; FRANCA, R.M.; VÁSQUEZ, E.M.F. \& FRISCHKORN, H. Avaliação da capacidade de infiltração de solos submetidos a diferentes tipos de manejo. Eng. Sanit. Amb., 14:89-98, 2009.

MERLIM, A.O.; GUERRA, J.G.M.; JUNQUEIRA, R.M. \& AQUINO, A.M. Soil macrofauna in cover crops of figs grown under organic management. Sci. Agríc., 62:57-61, 2005.

MUSCHLER, R.G. Árboles en cafetales. Turrialba, Costa Rica, CATIE/ GTZ, 2000. 139p.
OLIVEIRA, G.C.; DIAS JUNIOR, M.S.; RESCK, D.V.S. \& CURI, N. Caracterização química e físico-hídrica de um Latossolo Vermelho após vinte anos de manejo e cultivo do solo. R. Bras. Ci. Solo, 28:327-336, 2004.

REATTO, A.; BRUAND, A.; MARTINS, E.S.; MULLER, F.; SILVA, E.M.; CARVALHO JR., O.A.; BROSSARD, M. \& RICHARD, G. Development and origin of the microgranular structure in Latosols of the Brazilian Central Plateau: Significance of texture, mineralogy, and biological activity. Catena, 76:122-134, 2009.

SANTOS, M.L.; CARVALHO, M.P.; RAPASSI, R.M.A.; MURAISHI, C.T.; MALLER, A. \& MATOS, F.A. Correlação linear e espacial entre produtividade de milho (Zea mays L.) e atributos físicos de um Latossolo Vermelho distroférrico sob plantio direto do Cerrado Brasileiro. Acta Sci. Agron., 28:313-321, 2006.

SILVA, S.R.; BARROS, N.F. \& COSTA, L.M. Atributos físicos de dois Latossolos afetados pela compactação do solo. R. Bras. Eng. Agric. Amb., 10:842-847, 2006.

SOCARRÁS, A.V. La vida del suelo: un indicador de su fertilidad. Agric. Org., 4:12-14, 1998.

SOUZA, E.D.; CARNEIRO, M.A.C. \& PAULINO, H.B. Atributos físicos de um Neossolo Quartzarênico e um Latossolo Vermelho sob diferentes usos e manejos. Pesq. Agropec. Bras., 4:1135-1139, 2005.

SOUZA, Z.M. \& ALVES, M.C. Movimento de água e resistência à penetração em um Latossolo Vermelho distrófico de cerrado, sob diferentes usos e manejos. R. Bras. Eng. Agríc. Amb., 7:18-23, 2003.

STOLF, R.; FERNANDES, J. \& FURLANI NETO, V.L. Recomendações para uso de penetrômetro de impacto, modelo IAA/Planalsucar- Stolf. São Paulo, MIC/IAA/ PNMC - Planasulcar, 1983. 8p. (Série Penetrômetro de Impacto, BT1) 